\title{
vZ - An Optimizing SMT Solver
}

\section{Bjørner, Nikolaj; Dung, Phan Anh; Fleckenstein, Lars}

\section{Published in:}

Proceedings of the 21st International Conference on Tools and Algorithms for the Construction and Analysis of Systems, TACAS 2015

Link to article, DOI:

10.1007/978-3-662-46681-0_14

Publication date:

2015

Link back to DTU Orbit

Citation (APA):

Bjørner, N., Dung, P. A., \& Fleckenstein, L. (2015). vZ - An Optimizing SMT Solver. In Proceedings of the 21st International Conference on Tools and Algorithms for the Construction and Analysis of Systems, TACAS 2015 (pp. 194-199). Springer. Lecture Notes in Computer Science Vol. 9035 https://doi.org/10.1007/978-3-662-466810_14

\section{General rights}

Copyright and moral rights for the publications made accessible in the public portal are retained by the authors and/or other copyright owners and it is a condition of accessing publications that users recognise and abide by the legal requirements associated with these rights.

- Users may download and print one copy of any publication from the public portal for the purpose of private study or research.

- You may not further distribute the material or use it for any profit-making activity or commercial gain

- You may freely distribute the URL identifying the publication in the public portal 


\title{
$\nu Z$ - An Optimizing SMT Solver
}

\author{
Nikolaj Bjørner ${ }^{1}$, Anh-Dung Phan ${ }^{2}$, and Lars Fleckenstein ${ }^{3}$ \\ 1 Microsoft Research, Redmond, WA, USA \\ 2 DTU Compute, Technical University of Denmark \\ 3 Microsoft Dynamics \\ nbjorner@microsoft.com, padu@dtu.dk, LarsFleckenstein@outlook.com
}

\begin{abstract}
Z$ is a part of the SMT solver Z3. It allows users to pose and solve optimization problems modulo theories. Many SMT applications use models to provide satisfying assignments, and a growing number of these build on top of Z3 to get optimal assignments with respect to objective functions. $\nu Z$ provides a portfolio of approaches for solving linear optimization problems over SMT formulas, MaxSMT, and their combinations. Objective functions are combined as either Pareto fronts, lexicographically, or each objective is optimized independently. We describe usage scenarios of $\nu Z$, outline the tool architecture that allows dispatching problems to special purpose solvers, and examine use cases.
\end{abstract}

\section{An Invitation to $\nu Z$}

$\nu Z$ extends the functionality of Z3 [7] to include optimization objectives. It allows users to solve SMT constraints and at the same time formulate optimality criteria for the solutions. It relieves users of Z3 from writing their own loops around the solver to find optimal values. The solver integrates state-of-the-art algorithms for optimization, and it extends some of these algorithms with its own twists: For example, it includes direct support for difference logic solvers, it uses Simplex over non-standard numbers to find unbounded constraints, and it applies an incremental version of the MaxRes [11] algorithm for MaxSAT solving.

To give a first idea, we can ask to optimize the term $x+y$ under the constraints $y<5 \wedge x<2$ and $y-x<1$ using the SMT query to the right. The optimal answer is given as 2 and $\nu Z$ returns a model where $x=y=$ 1. The example shows the maximize command that is added to the SMT-

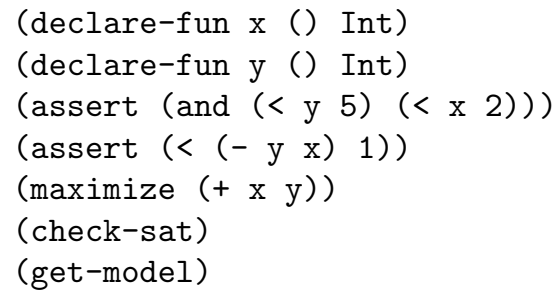

\subsection{Optimization Commands}

The full set of commands $\nu Z$ adds to SMT-LIB are: 


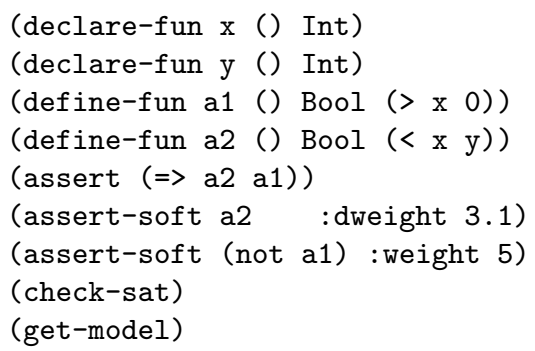

Fig. 1. Maximize $3.1 \cdot a 2+5 \cdot \overline{a 1} . \nu Z$ finds a solution where $y \leq x \leq 0$.

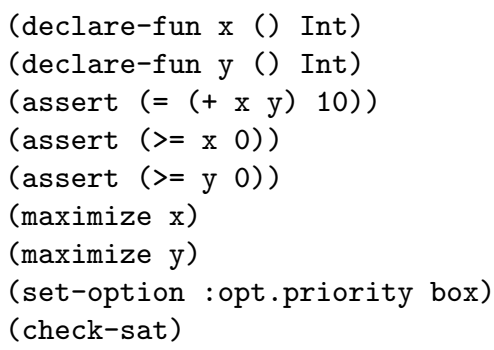

Fig. 2. $\nu Z$ produces two independent optima $x=10$, respectively $y=10$.

- (maximize $t$ ) - instruct the solver to maximize $t$. The type of the term $t$ can be either Integer, Real or Bit-vector.

- (minimize $t$ ) - instruct the solver to minimize $t$.

- (assert-soft $F$ [:weight $n \mid$ :dweight $d]\left[\begin{array}{ll}: i d & i d\end{array}\right]$ ) - assert soft constraint $F$, optionally with an integral weight $n$ or a decimal weight $d$. If no weight is given, the default weight is 1 (1.0). Decimal and integral weights can be mixed freely. Soft constraints can be furthermore tagged with an optional name $i d$. This enables combining multiple different soft objectives. Fig. 1 illustrates a use with soft constraints.

\subsection{Combining Objectives}

Multiple objectives can be combined using lexicographic, Pareto fronts or as independent box objectives.

Lexicographic combinations: By default, $\nu Z$ maximizes objectives $t_{1}, t_{2}$ subject to the constraint $F$ using a lexicographic combination. It finds a model $M$, such that $M$ satisfies $F$ and the pair $\left\langle M\left(t_{1}\right), M\left(t_{2}\right)\right\rangle$ is lexicographically maximal. In other words, there is no model $M^{\prime}$ of $F$, such that either $M^{\prime}\left(t_{1}\right)>M\left(t_{1}\right)$ or $M^{\prime}\left(t_{1}\right)=M\left(t_{1}\right), M^{\prime}\left(t_{2}\right)>M\left(t_{2}\right)$.

Pareto fronts: Again, given two maximization objectives $t_{1}, t_{2}$, the set of Pareto fronts under $F$ are the set of models $M_{1}, \ldots, M_{i}, \ldots, M_{j}, \ldots$, such that either $M_{i}\left(t_{1}\right)>M_{j}\left(t_{1}\right)$ or $M_{i}\left(t_{2}\right)>M_{j}\left(t_{2}\right)$, and at the same time either $M_{i}\left(t_{1}\right)<$ $M_{j}\left(t_{1}\right)$ or $M_{i}\left(t_{2}\right)<M_{j}\left(t_{2}\right)$; and for each $M_{i}$, there is no $M^{\prime}$ that dominates $M_{i}$. $\nu Z$ uses the Guided Improvement Algorithm [14] to produce multiple objectives. Fig. 3 illustrates a use where Pareto combination is specified.

Boxes: Box objectives, illustrated in Fig 2 are used to specify independent optima subject to a formula $F$. They are used in the Symba tool [9]. The box combination of objectives $t_{1}, t_{2}$ requires up to two models $M_{1}, M_{2}$ of $F$, such that $M_{1}\left(t_{1}\right)$ is the maximal value of $t_{1}$ and $M_{2}\left(t_{2}\right)$ is the maximal value for $t_{2}$. 


\subsection{Programming Optimization}

The optimization features are available over Z3's programmatic APIs for C, $\mathrm{C}++$, Java, .NET, and Python. There is furthermore a library available as an example that plugs into the Microsoft Solver Foundation (MSF). Fig. 3 shows an example using the Python API to generate Pareto optimal solutions. Fig. 4 shows an OML model used by MSF.

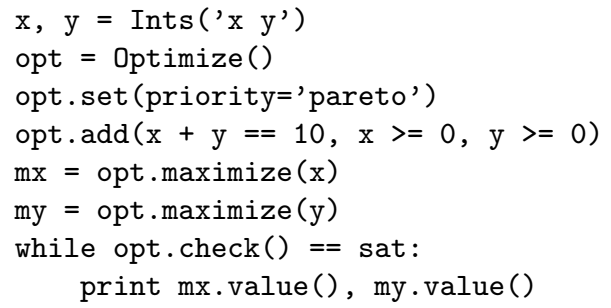

Fig. 3. Pareto optimization in Python. $\nu Z$ produces all 11 Pareto fronts.

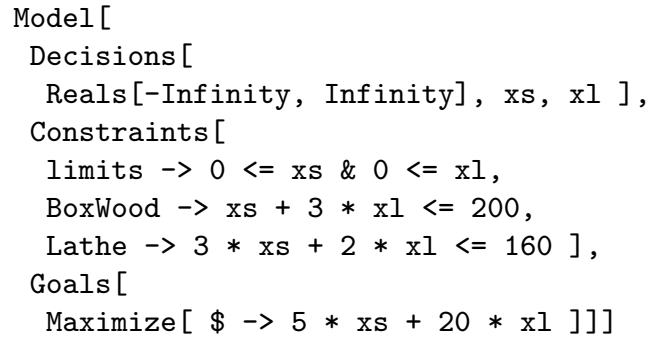

Fig. 4. OML model used by MSF.

\subsection{MILP, MaxSAT, CP and SMT}

Efficient mixed integer linear solvers are backbones of several highly tuned tools, such as CPLEX and Gurobi, used in operations research contexts. Being able to state and solve optimization objectives in the context of logical constraints has also been well recognized in the SMT community [12|5|15|8 and it is a recurring feature request for Z3 as well. We briefly outline a use case in Section 4, and through this experience we observed a need for more abstract and flexible ways of modeling problems than exposed by OML used by the Microsoft Solver Foundation (MSF), where flexible Boolean combinations of constraints, which empower end-users to refine models, are afterthoughts. By making $\nu Z$ generally available, we hope to make it easier for existing users to use Z3, for instance [2], and to fuel further applications that benefit from the flexibility and expressive power of Z3's SMT engines, including theory support and quantifiers, with the convenience of built-in support for (reasonably tuned) optimization algorithms. In return, we anticipate that new applications from SMT users can inspire advances in areas such as non-linear arithmetic, mixed symbolic/numerical algorithms, and combinations with Horn clauses.

\subsection{Resources}

The full source code of $\nu Z$ is available with Z3 from http://z3.codeplex.com, the sources compile on all main platforms, there is an online tutorial on http:// rise4fun.com/z3opt/tutorial/, and a companion paper [3] describes details of algorithms used in $\nu Z$. 


\section{Architecture}

Fig. 5 gives an architectural overview of $\nu Z$. The input SMT formulas and objectives are rewritten and simplified using a custom strategy that detects 0-1 integer variables and rewrites these into Pseudo-Boolean Optimization (PBO) constraints. Objective functions over $0-1$ variables are rewritten as MaxSAT problems ${ }^{4}$. If there are multiple objectives, then $\nu Z$ orchestrates calls into the SMT or SAT cores. For box constraints over reals, $\nu Z$ combines all linear arithmetic objectives and invokes a single instance of the OptSMT engine; for lexicographic combinations of soft constraints, $\nu Z$ invokes the MaxSAT engine using multiple calls.

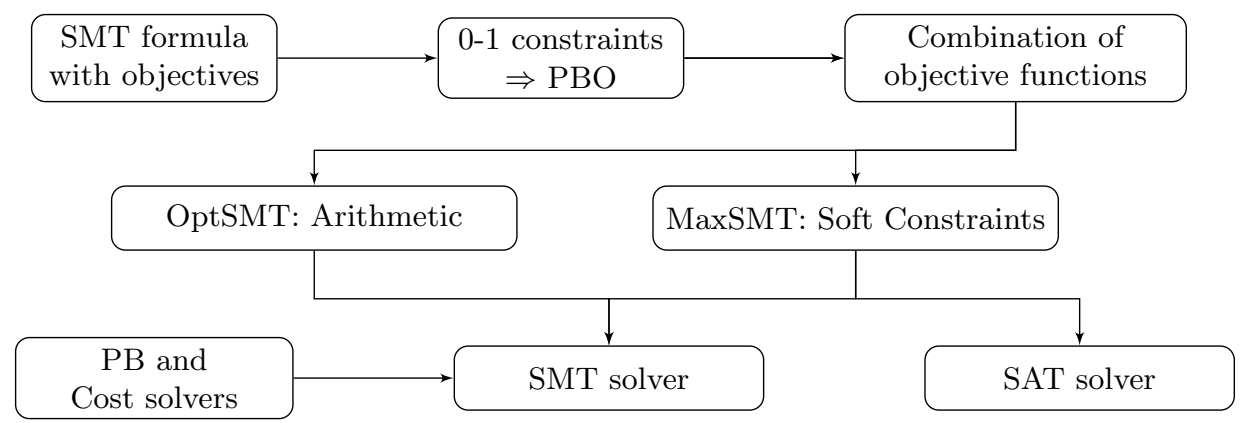

Fig. 5. $\nu Z$ system architecture

\section{Internals}

OptSMT: We have augmented Z3's dual Simplex core with a primal phase that finds maximal assignments for reals. It also improves bounds on integers as long as the improvements are integral. It is used, similarly to [15]9, to improve values of objective functions. A similar primal Simplex solver is also accessible to Z3's difference logic engines. $\nu Z$ discovers unbounded objectives by using non-standard arithmetic: It checks if $t \geq \infty$ is feasible, over the extension field $R \cup\{\epsilon, \infty:=1 / \epsilon\}$. This contrasts the approach proposed in 9 that uses a search through hyper-planes extracted from inequalities.

$\nu Z$ also contains a Pseudo-Boolean theory solver. It borrows from [4] for simplification, generating conflict clauses, and incrementally compiling into small sorting circuits. It also adds an option to prune branches using dual simplex.

MaxSMT: $\nu Z$ implements several engines for MaxSAT. These include WMax [12], MaxRes [1], BCD2 [10, MaxHS [6]. WMax uses a specialized theory solver of

${ }^{4}$ using the correspondence: maximize $c_{1} \cdot x_{1}+c_{2} \cdot x_{2} \equiv$ (assert-soft $x_{1}$ :weight $\left.c_{1}\right)$, (assert-soft $x_{2}$ :weight $c_{2}$ ) 
costs, also explored in [5]. The solver associates penalties with a set of tracked propositional variables. It then monitors the truth assignments to these variables, as given by the SAT solver. The cost is incremented when a tracked variable is assigned to false. The solver creates a conflict clause when the cost exceeds the current optimal value. WMax can be interrupted at any point with a current upper bound. Our implementation of MaxRes generally performs much better than WMax. MaxRes increments a lower bound when there is an unsatisfiable core of the soft constraints. It then replaces the core $F_{1}, \ldots, F_{k}$ with new soft constraints $F_{1}^{\prime}, F_{2}^{\prime}, \ldots, F_{k-1}^{\prime}$ using the equations:

$F_{1}^{\prime}=F_{2} \vee F_{1}, F_{2}^{\prime}=F_{3} \vee\left(F_{1} \wedge F_{2}\right), \ldots, F_{k-1}^{\prime}=F_{k} \vee\left(\left(F_{1} \wedge F_{2}\right) \wedge \ldots \wedge F_{k-1}\right)$

SAT: $\nu Z$ reduces Pseudo-Boolean formulas to propositional SAT by converting cardinality constraints using sorting circuits, using a Shannon decomposition (BDDs) of simple PB inequalities and falling back to bit-vector constraints on inequalities where the BDD conversion is too expensive. This transformation is available by ensuring that the option : opt.enable_sat is true. For benchmarks that can be fully reduced to propositional SAT, MaxRes uses Z3's SAT solver.

\section{A use for $\nu Z$}

As a driving scenario for $\nu Z$ we used an experimental warehouse manager in the context of Microsoft Dynamics AX. The objective is to reduce cost by optimizing how shipments are distributed on trucks, reducing the number of trucks, the distance traveled by the truck while maximizing the amount of goods delivered. AX can deliver the standard constraints and cost functions, e.g., weight and volume of a truck, but users often want to be more specific. For example, frozen foods need to be in a cooled truck and cannot be packed together with chemicals. The expressive power and convenience of SMT is useful: these constraints can be formulated as a Boolean combination of linear constraints over 0-1 variables, while the objective functions we considered could be expressed as lexicographic combinations of a couple of cost functions. Such cost functions are expected to evolve when users learn more about their usages. The abstraction layer of the models provides this flexibility.

\subsection{Experience}

We evaluated $\nu Z$ on a cross-section of benchmarks used in MaxSAT competitions, from Z3 users, and from recent publications. Table 1 summarizes a selected evaluation. Motivating examples from users included strategy scheduling for Vampire (MaxSAT) that are easy with the new MaxSAT engine, but used to be hard for the bisection search used by Vampire. Likewise, Cezary Kaliszyk has used Z3 to tune his portfolio solver using linear arithmetic constraints. His systems are significantly more challenging (take days to run). In this case WMax offers partial solutions during search. Elvira Albert tried using Z3 for finding 
longest paths, her benchmarks are called bb $(\approx 300$ clauses $)$, chat $(\approx 3 \mathrm{~K}$ clauses $)$ and $\mathrm{p} 2 \mathrm{p}(\approx 30 \mathrm{~K}$ clauses $)$, and we summarize timing for bb and chat below. The p2p category remains really hard. Korovin and White experimented with encoding RNA alignment into MaxSAT, and Pandya and Bresler encoded DNA alignment problems into MaxSMT over LIA. Many problems remain challenging, including material discovery benchmarks from Ronan Le Bras, Pareto problems from Oded Maler and even benchmarks from the pure Pseudo-Boolean domain 5 .

\begin{tabular}{|c|c|c|c|}
\hline Source & Category & Solved instances & Time \\
\hline MaxSAT 2014 wpms industrial track & MaxSAT & $361 / 410$ & $0.5-1800 \mathrm{~s}$ \\
\hline MaxSAT 2014 pms industrial track & MaxSAT & $406 / 568$ & $0.5-1800 \mathrm{~s}$ \\
\hline Longest Paths & MaxSAT & bb $8 / 8$ & $<0.05 \mathrm{~s}$ \\
\hline Longest Paths & MaxSAT & chat $34 / 34$ & $1-36 \mathrm{~s}$ \\
\hline DAL Allocation challenge & $\mathrm{PBO}$ & SampleA\&B 96/96 & $0.02-6 \mathrm{~s}$ \\
\hline Symba $[9]$ & LRA & $2435 / 2435$ & $0.2 \mathrm{~s}-36 \mathrm{~s}$ \\
\hline OptiMathSAT 15 & LRA & 9 non-random & $0.5-20 \mathrm{~s}$ \\
\hline
\end{tabular}

Table 1. Evaluation of $\nu Z$ on selected examples

\section{References}

1. I. Abío, R. Nieuwenhuis, A. Oliveras, and E. R.-Carbonell. A parametric approach for smaller and better encodings of cardinality constraints. In $C P, 2013$.

2. K. Becker, B. Schätz, M. Armbruster, and C. Buckl. A formal model for constraintbased deployment calculation and analysis for fault-tolerant systems. In $S E F M$, pages 205-219, 2014.

3. N. Bjørner and A.-D. Phan. $\nu Z$ - Maximal Satisfaction with Z3. In SCSS, 2014.

4. D. Chai and A. Kuehlmann. A fast pseudo-boolean constraint solver. IEEE Trans. on CAD of Integrated Circuits and Systems, 24(3):305-317, 2005.

5. A. Cimatti, A. Franzén, A. Griggio, R. Sebastiani, and C. Stenico. Satisfiability modulo the theory of costs: Foundations and applications. In TACAS, 2010.

6. J. Davies and F. Bacchus. Postponing optimization to speed up MAXSAT solving. In $C P$, pages $247-262,2013$.

7. L. M. de Moura and N. Bjørner. Z3: An Efficient SMT Solver. In TACAS, 2008.

8. D. Larraz, K. Nimkar, A. Oliveras, E. Rodríguez-Carbonell, and A. Rubio. Proving Non-termination Using Max-SMT. In CAV, pages 779-796, 2014.

9. Y. Li, A. Albarghouthi, Z. Kincaid, A. Gurfinkel, and M. Chechik. Symbolic optimization with SMT solvers. In POPL, pages 607-618, 2014.

10. A. Morgado, F. Heras, and J. Marques-Silva. Improvements to Core-Guided Binary Search for MaxSAT. In SAT, pages 284-297, 2012.

11. N. Narodytska and F. Bacchus. Maximum Satisfiability Using Core-Guided MaxSAT Resolution. In AAAI, pages 2717-2723, 2014.

12. R. Nieuwenhuis and A. Oliveras. On SAT Modulo Theories and Optimization Problems. In SAT, pages 156-169, 2006.

13. S. Ranise and C. Tinelli. The SMT Library (SMT-LIB). www.SMT-LIB.org, 2006.

14. D. Rayside, H.-C. Estler, and D. Jackson. The Guided Improvement Algorithm. Technical Report MIT-CSAIL-TR-2009-033, MIT, 2009.

15. R. Sebastiani and S. Tomasi. Optimization in SMT with $\mathcal{L} \mathcal{A}(\mathbf{Q})$ Cost Functions. In IJCAR, pages 484-498, 2012.

\footnotetext{
5 http://www.cril.univ-artois.fr/PB12/
} 


\section{A Demonstration}

We take a quick tour on available features in $\nu Z$. This section is based on the online tutorial at http://rise4fun.com/Z30pt/tutorial/guide. Please visit the online playground for a quick try at $\nu Z$. More details of internals in $\nu Z$ are also available in a paper [3] that accompanies an invited talk at SCSS 2014. The paper describes, at a high level, the algorithms used for different optimization strategies in $\nu Z$.

\section{Linear Arithmetic}

Using the example in Fig. 2, $\nu Z$ returns two independent values 10 and 10 for two objectives $x$ and $y$.

\section{3opt ${ }^{\text {Research }}$ z3opt}

What is an optimal model for this formula?

1 (declare-fun $x$ () Int)

2 (declare-fun y () Int)

3 (assert $(=(+x y) 10))$

4 (assert $(>=x \theta)$ )

5 (assert $(>=y \quad \theta)$ )

6 (maximize $\mathrm{x}$ )

7 (maximize $y$ )

8 (set-option :opt.priority box)

9 (check-sat)
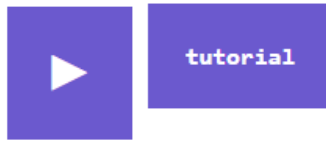

$$
\begin{aligned}
& x \mid->10 \\
& y \quad \mid->10 \\
& \text { sat }
\end{aligned}
$$

This example can be reproduced at http://rise4fun.com/Z30pt/o7h.

\section{Soft Constraints}

The example in Fig. 1 demonstrates soft constraints with integral and decimal weights. $\nu Z$ gives an optimal model where the total weight equals to 3.1 and $x=y=0$. 


\section{z3opt ${ }^{\text {Research }}$}

What is an optimal model for this formula?

1 (declare-fun $x$ () Int)

2 (declare-fun y () Int)

3 (define-fun a1 () Bool (> $\times$ ( $\theta)$ )

4 (define-fun a2 () Bool $(<x y)$ )

5 (assert ( $\Rightarrow$ a2 a1))

6 (assert-soft a2 :dweight 3.1)

7 (assert-soft (not a1) :weight 5)

8 (check-sat)

9 (get-model)

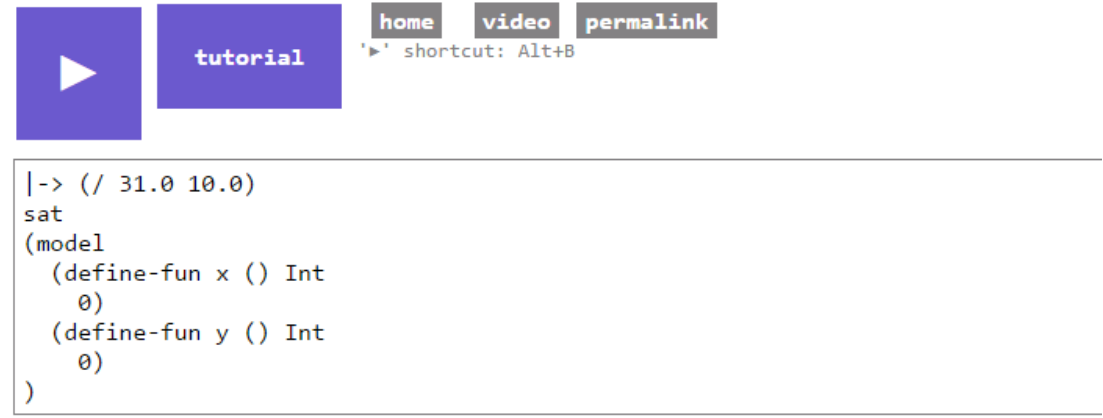

This example can be reproduced at http://rise4fun.com/Z30pt/GR.

\section{Programmatic APIs}

$\nu Z$ 's APIs are available for multiple languages. This section presents programmatic APIs using a few F\# code snippets.

Optimization features are facilitated through the new Optimize solver. As being shown in Fig. 6, combining objectives can be set via solver parameters. MkMinimize and Value are used to set optimization goals and retrieve optimal values respectively (see Fig. 7).

PB constraints are available via MkAtMost and MkPBLe commands (see Fig. 8). They allow users to express some problems in a more concise manner. Soft constraints can be sent to the solver using the AssertSoft command. As Fig. 7 demonstrated, users can employ Check and Value methods for getting optimal values. 
to run multiple solvers in parallel with $\nu Z$ inside MSF. The plugin plays a vital role in bootstrapping $\nu Z$ by comparing it with other solvers in MSF.

\section{Case study}

In this section, we use the scenario of a warehouse manager to demonstrate how $\nu Z$ can be used for solving problems inspired by Dynamics AX scenarios. Suppose that there are a number of goods that need to be shipped to customers. These goods have specific destinations and requirements as shown in Table 2 .

\begin{tabular}{|l|l|l|l|l|}
\hline Shipment no. & Weight $(\mathrm{kg})$ & Volume $\left(\mathrm{m}^{3}\right)$ & Requirement & Zip code \\
\hline \hline 1 & 400 & 300 & Dry & 2112 \\
\hline 2 & 300 & 350 & Fresh & 2100 \\
\hline 3 & 220 & 160 & Dry & 2103 \\
\hline
\end{tabular}

Table 2. Shipments that requires transportation.

Table 3 describes trucks along with their capacities, tentative destinations and associated costs. The constraints are:

1. Shipments have to fit in the capacities given by the trucks.

2. Product requirements have to be fulfilled.

3. Shipments are delivered to correct addresses (zip codes).

We can state the goals in their order of priorities (1) Use as few trucks as possible (2) Minimize the total cost for transportation. The scenario is a variant of a bin packing problem. See also the ESICUP Challenge.

\begin{tabular}{|l|l|l|l|l|l|}
\hline Truck no. & Weight $(\mathrm{kg})$ & Volume $\left(\mathrm{m}^{3}\right)$ & Requirement & Zip codes & Initial cost $(\mathrm{USD})$ \\
\hline \hline 1 & 777 & 700 & Fresh & 2100,2103 & 100 \\
\hline 2 & 450 & 1000 & Dry & $2100,2103,2112$ & 120 \\
\hline 3 & 600 & 460 & Dry & 2100,2112 & 130 \\
\hline
\end{tabular}

Table 3. Available trucks for transportation.

The constraints and goals can be modeled directly as $0-1$ variables. Let the 0-1 variable $x_{i j}$ denote that shipment $i$ is packed into truck $j$. Each shipment has to be in exactly one truck.

Let $0-1$ variable $y_{j}$ denote that truck $j$ is in use. Trucks are used if something is packed into them:

Here we describe weight as examples of capacity constraints. There are similar constraints for volumes.

To keep this example simple we here treat zip codes as fixed costs. The goals can be modeled as in Fig. 14. Note that we would like to minimize the number 


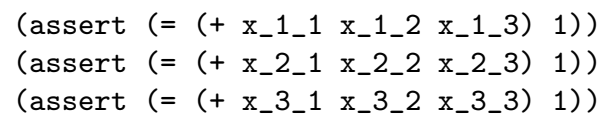

Fig. 11. Each shipment is in only one truck.

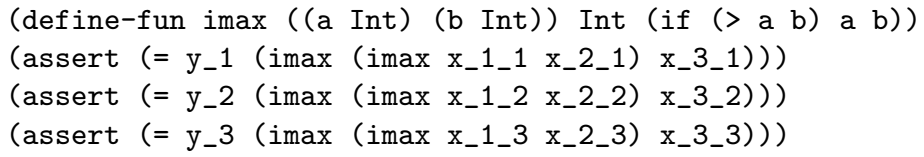

Fig. 12. Truck $j\left(y_{j}\right)$ is in use.

of used trucks and minimize total cost, so in MaxSMT these goals are equivalent to maximizing number of unused trucks and maximizing saving.

Although we express the problem via $0-1$ variables, $\nu Z$ translates it via Pseudo-Boolean constraints (opt.elim_01=true by default). In this case $\nu Z$ uses the Pseudo-Boolean solver and solves a MaxSMT problem. Furthermore, by ensuring that opt.enable_sat $=$ true, opt.maxsat_engine $=$ maxres, $\nu Z$ translates Pseudo-Boolean constraints to SAT and uses a pure MaxSAT solver.

The example illustrates a fair amount of flexibility in formulating constraints, and control over the most suitable back-end for these constraints.

\section{Selected evaluation details}

In Fig. 15, the two top graphs show comparison with $\nu Z$ and the best solvers in MaxSAT 2014 competition. Note that $\nu Z$ is presently not fine-tuned as some of these competition entrants are, but performs quite respectably. What is not shown is performance of other solvers entering the competition and performing on par or worse than $\nu Z$. The bottom graph shows relative comparison of $\nu Z$ with and without the Symba approach of finding unbounded objectives. The experience indicates that searching separately for unbounded objectives does not really help.

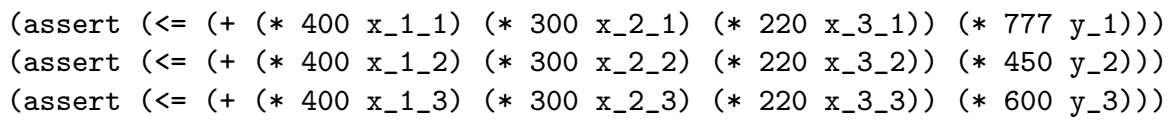

Fig. 13. Capacity constraints. 


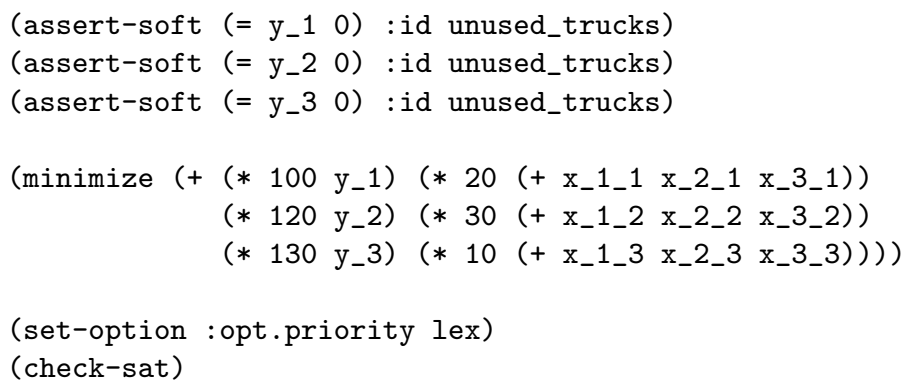

Fig. 14. Two optimization goals of the truck planning example.
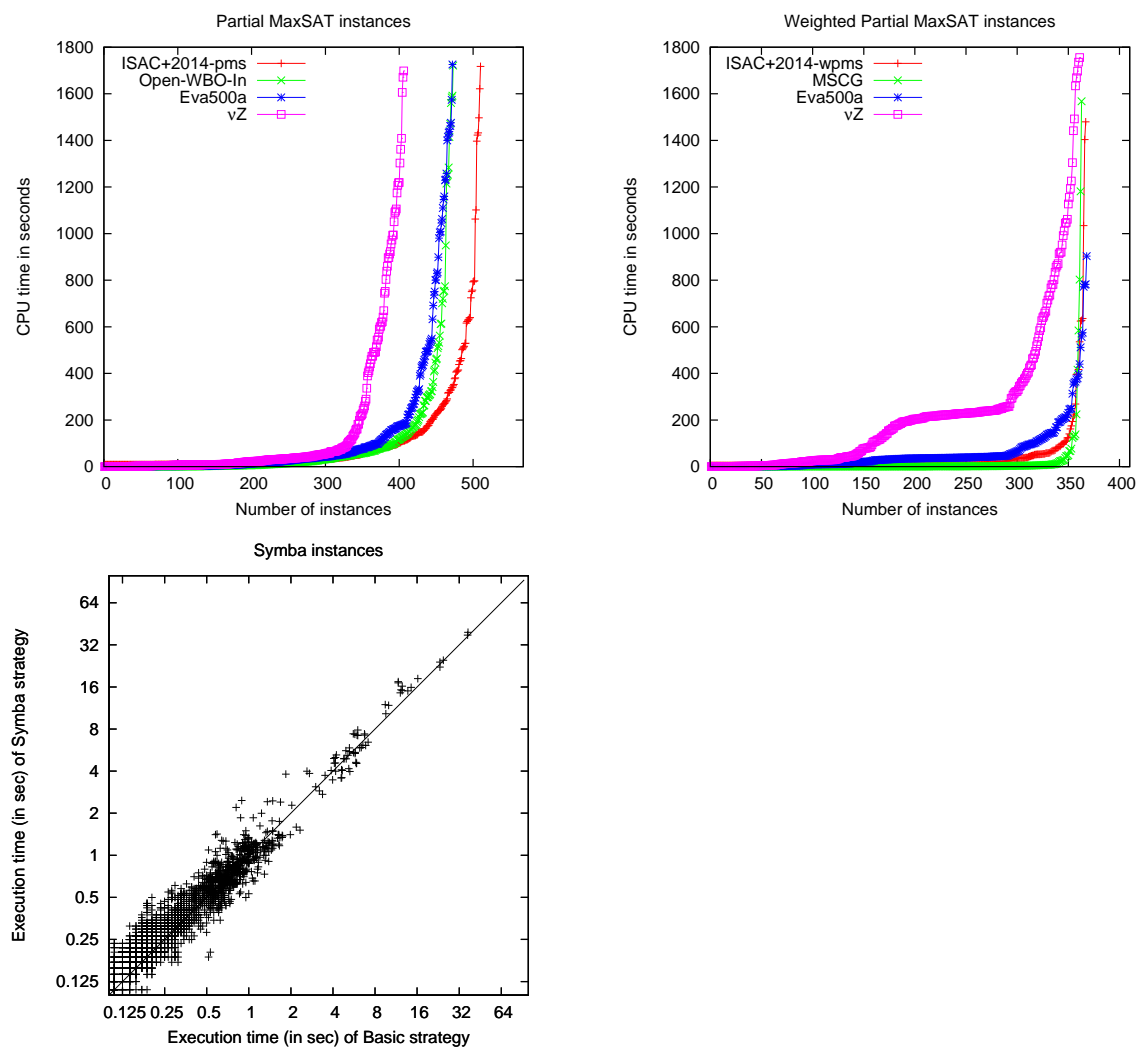

Fig. 15. Evaluation results. 